\title{
Identification of Some Oat Cultivars (Avena sativa L.) By Uusing Morphological Characters of $T$ the Spikes
}

\author{
A. A. dalal Bashi \\ Department of biology \\ Girls Education College \\ University of Mosul \\ Amina.dlalalbashi@gmail.com
}

\section{DOI: $\underline{10.33899 / \text { edusj.2019.162971 }}$}

\section{Received \\ 24 / 12 / 2018}

\author{
A. S. Jabar \\ Department of biology \\ Education College \\ University of Mosul \\ Dr.aziz49@yahoo.com
}

Accepted
$27 / 02 / 2019$

\begin{abstract}
This study was conducted in the wired house and the laboratories of the Department of Biology, College of Education, Mosul University, in the period extended from 1/4/2009 until 1/11/2009 to study many morphological characters of all the five imported cultivates of oat plants (Possum 6, Kangaroo 4, Oat Tall, Mittika 5, Oat Short). The results of this study were statistically analyzed according to Complete Randomized Design. The statistical results were considered significant at $\mathrm{P} \leq 0.05$. The results showed significant differences in many of the morphological characters of all the five cultivars including length and shape of rachis, peduncle length, pedicle length, Arrangement of spike, Glumes length, Hairs on the lemma, Awn length, Ligules height and shape.

In conclusion; it is possible to identify and classify these five Oats cultivars by studying the morphological characters of its spikes.
\end{abstract}

Key word: Oat, Morphological characters, spikes, Pedicle 
تشخيص بعض أصناف الثوفان .Avena sativa L المستوردة باستخدام الصفات المظهرية للسنابل

\author{
عبد العزيز شيخو عبد الجبار \\ قسم علوم حياة / كلية التربية

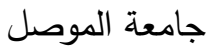 \\ Dr.aziz49@yahoo.com
}

\author{
امنة احمد يحيى دلال باشي \\ قسم علوم حياة / كلية التربية للبنات

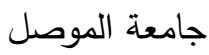 \\ amina.dlalallbashi@ gmail.com
}

DOI: $\underline{10.33899 / \text { edusj.2019.162971 }}$

القبول

2019 / 02 / 27
الاستلام

2018 / 12 / 24

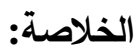

تم اجراء هذه الدراسة في البيت السلكي والمختبرات التابع لقسم علوم الحياة في كلية التربية/ جامعة الموصل للمدة بين 2009/10/25 ولغايـة 2010/3/25 ـ وقد شمل البحث دراسـة الصفات المظهريـة لسنابل خمسة أصناف زراعية من نبات الشوفان Avena sativa المستوردة وهي (1) Kangaroo 4 , Possum , ته


النتائج وجود اختلافات معنوية بين الاصناف الزراعية المدروسة في العديد من الصفات المظهرية وهي (طول

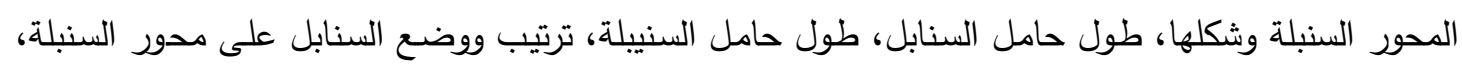
طول القنابع، وجود شعيرات على قاعدة العصيفة، طول السفا، طول او ارتفاع اللسين وشكل اللسين). يستتنج من هذه الدراسة وجود تباين في العديد في الصفات المظهرية لسنابل الاصناف الزراعية المدروسة.

الكلمات المفتاحية: الشوفان، الصفات المظهرية، السنابل، السنيبلة

المقدمة:

يعد علم تصـنيف النباتـات من اهم العلوم التطبيقيـة الحديثة في معرفـة الانواع والاصـناف الزراعيـة

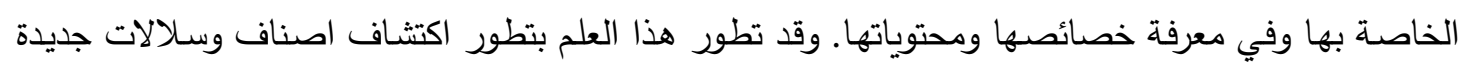

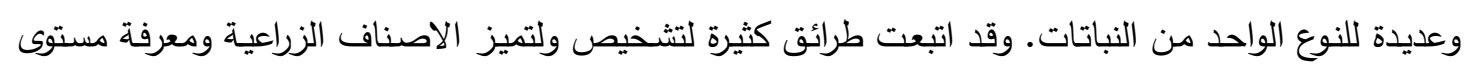

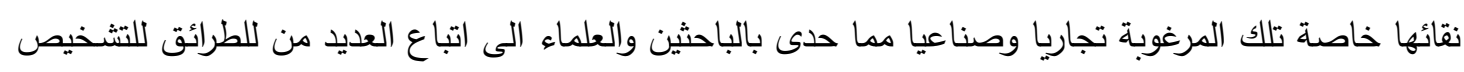

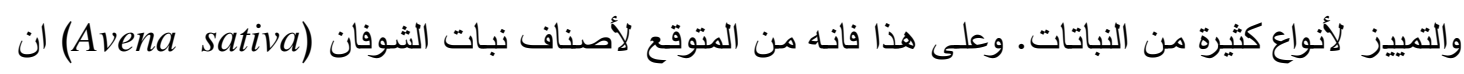

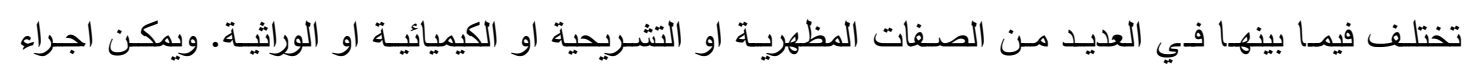
فحوصات معتمدة في الحقل او في المختبر لمعرفة هذه الفروقات وتتبيتها، [1] والتي تعد داعمة ومكملة للعديد

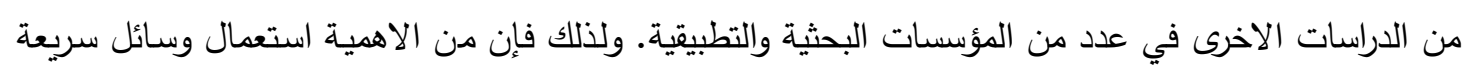

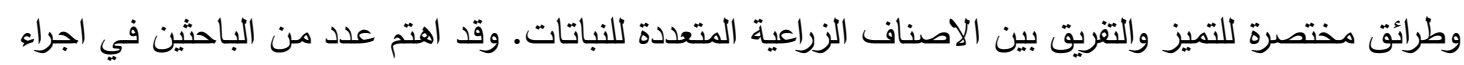

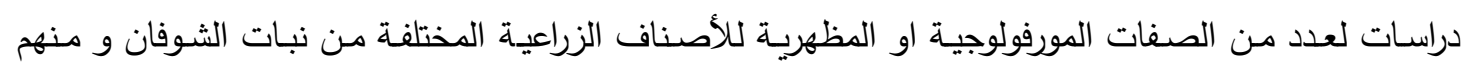


[2]،[3]،[4]،[5]. ويعد الشوفان من النباتات العشبية الحولية الذي ينتمي الى العائلة النجيلية والتي تتنج الحبوب

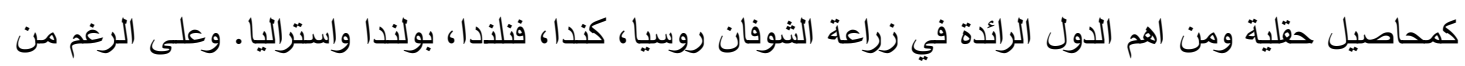

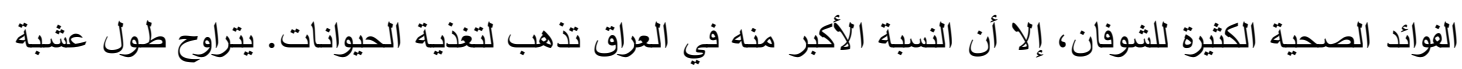

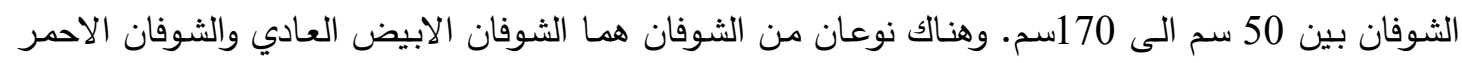

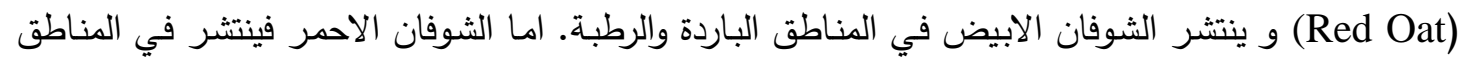

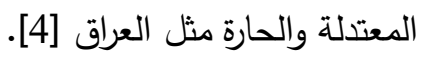
يعتبر الشوفان من النباتات التي تستعمل في غذاء الانسان والحيوان، فحبوب الشوفان غنية بالعناصر الغذائية المهمة كالبروتينات والكربوهيدرات والسعرات الحرارية والالياف والمعادن والفيتامينات [5]. يعود جنس Avena الى عائلة (Gramineae) (Grass family) التابعة لرتبة وتعد العائلة التي ينتمي لها الشوفان من اكبر العائلات النباتية اذ تتضمن هذه العائلة

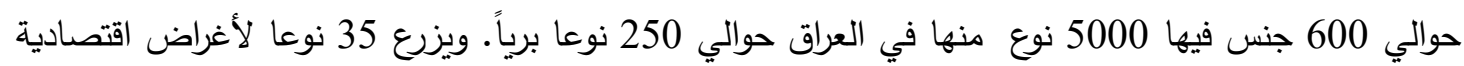

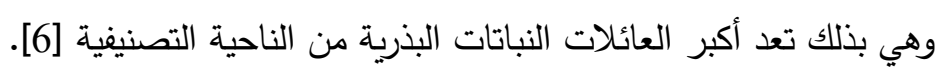
ويعتمد في تشخيص هذه العائلة على خصائص السنيبلات اكثر مما على الازهار اذ يمكن الاعتماد على حجم وشكل وقوام وتعرق قنابات السنيبلة (القنابع) وعلى وجود السفا وانعدامها وعدد الزهيرات في السنيبلة الواحدة [6]،[7]. ويهدف البحث الحالي الى دراسة الصفات المظهرية لخمسة من اصناف الثوفان العادي المستورد الى العراق لتثبيت صفاتها والحفاظ على الاصناف الزراعية المميزة منها واستعمل الصفات المظهريـة للنبات للتمييز

Materials and Methods المواد وطرائق العمل شملت هذه الدراسة خمسة اصناف من نبات الثوفان Avena sativa المستوردة من استراليا هي (Oat Short ,Mittika 5 ,Oat Tall , Kangaroo 4, Possum 6) الزراعية من المركز العالمي للبحوث الزراعية في المناطق الجافة (ICARDA) حلب/سوريا، والتي كانت قد ارسلت إلى قسم الفحص وتصديق البذور في محافظة نينوى عام (2009). وقد اجرينا المستلزمات الحقلية لزراعة

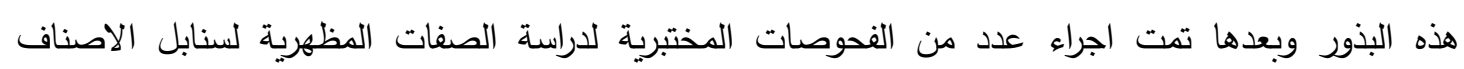
الزراعية قيد الدراسة.

Field works الاراسات الحقلية

تمت زراعة الحبوب للأصناف الزراعية الخمسة من الثوفان قيد الدراسـة خـلال الموسم الثتوي للعام

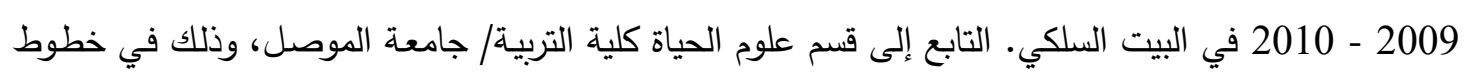

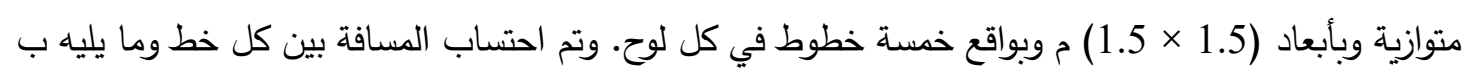



وبالتصعيم العشوائي الكامل (Completely Randomized Design).

ابتدأت زرعة البذور يدوياً بتاريخ 2009/11/4 وبموجب وحدة المساحة بما يعادل 25 كغم من البذور /

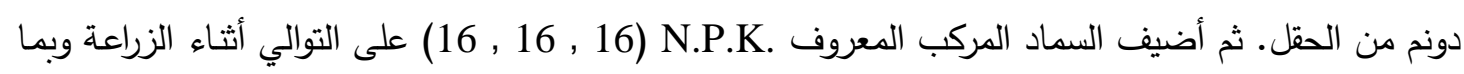
يعادل 25 كغم من السماد/ دونم من الحقل، وتمت المتابعة المناسبة للري وللتعشيب خلال فترة الزراعة والنمو. تم الانتهاء من فترة النمو واخذت العينات من النباتات المزروعة بتاريخ 20 /4 / 2010 / 
نباتات لكل مكرر و10 نباتات لكل صنف وبطريقة عشوائية (الشكل 1)، وتمت دراسة الصفات المظهرية لجميع الاصناف الزراعية المدروسة وكما يأتي:

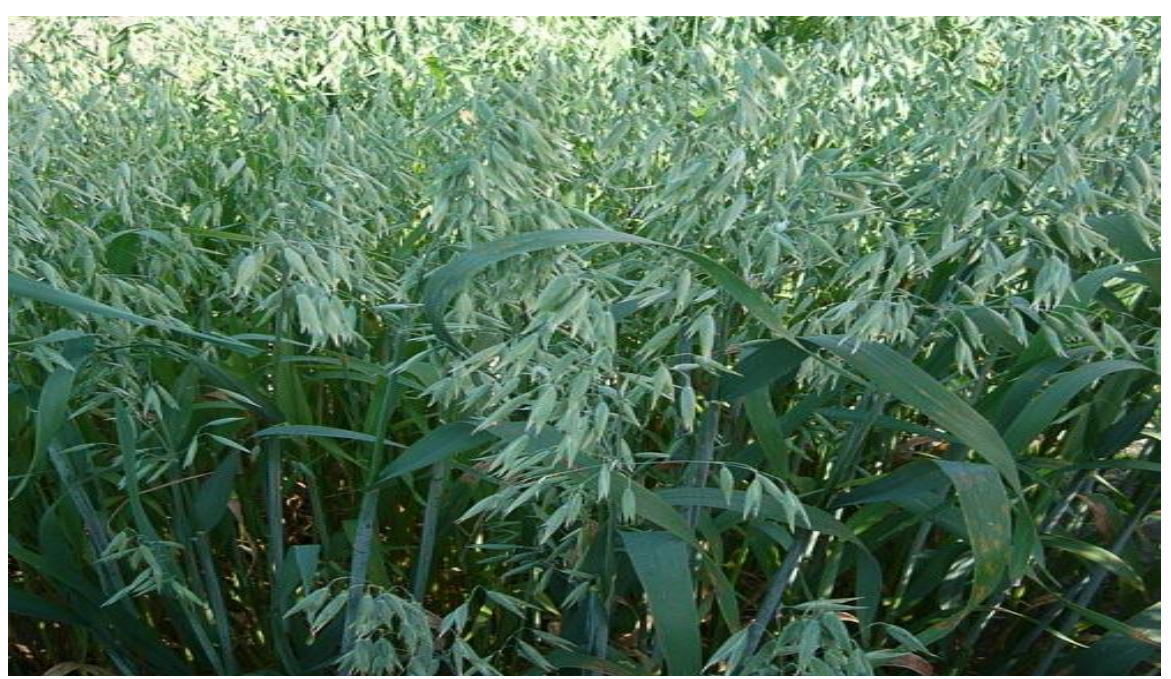

(أ) قبل مرحلة النضج

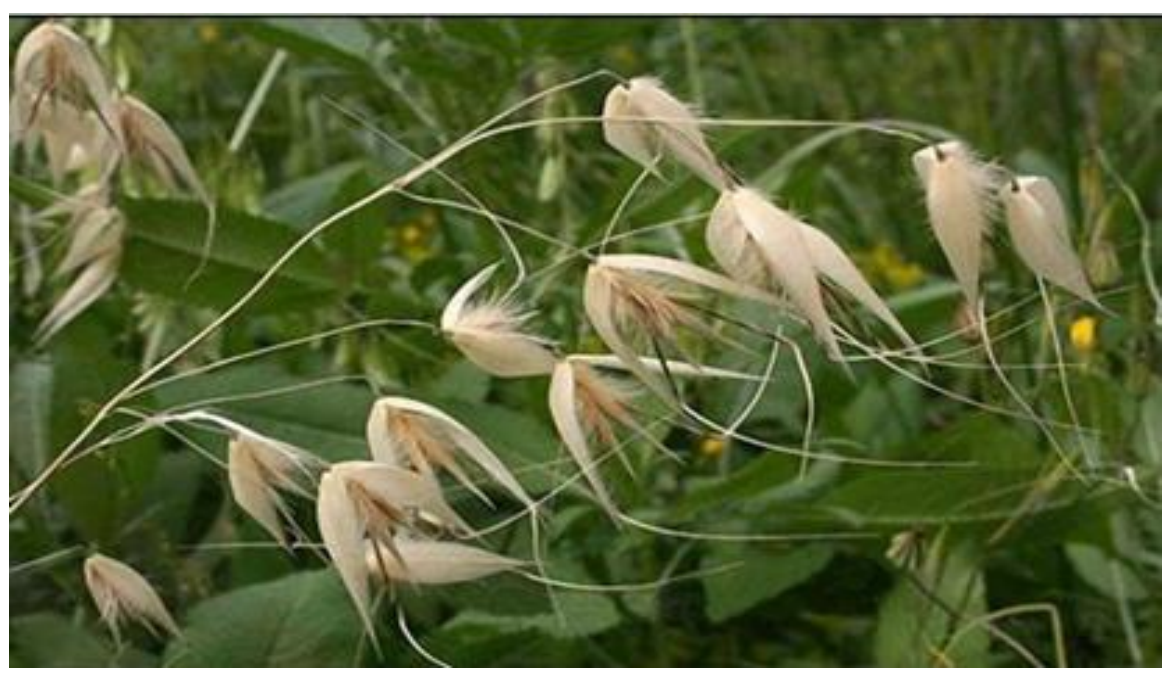

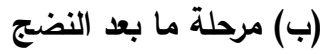

شكل (1): تبات الثوفان



استعملت مسطرة صغيرة مدرجة بالملمترات لاحتساب طول محور السنبلة وباتباع طريقة [8] (شكلة2).

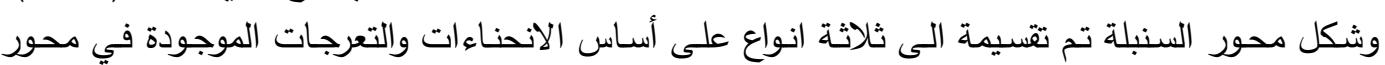
السنبلة (شكل 3) وكما يأتي: متعرج، قليل التعرج، غير متعرج.

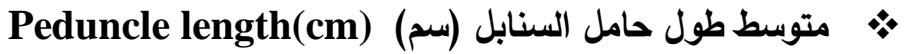
تم استعمال نفس المسطرة نفسها المذكورة لقياس طول تفرعات السنبلة كما في ( شكل 2) وبموجب طريقة

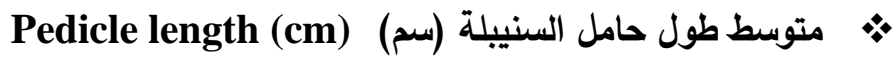
(تم استعمال ذات المسطرة المذكورة لحساب طول حامل السنيبلة (شكل 2) حسب طريقة [8]). 
Arrangement of spike on the Rachis ترتيب السنابل على محور السنبلة قسم وحسب طريقة [9] ترتيب السنابل على محور السنبلة على ثلاثة أقسام كما ياتي:

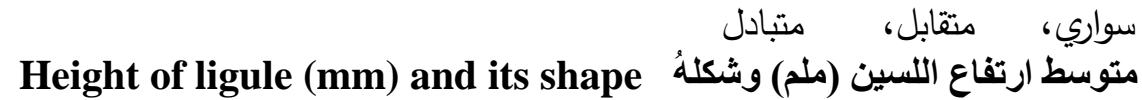



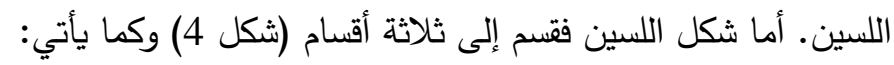

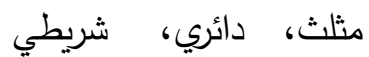

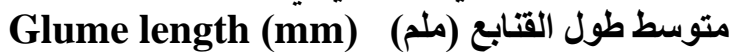
(تم استعمال المسطرة المذكورة انفا لاحتساب طول القنابع كما في (شكل 5) وحسب (1)]). Awn length متوسط طول السفال

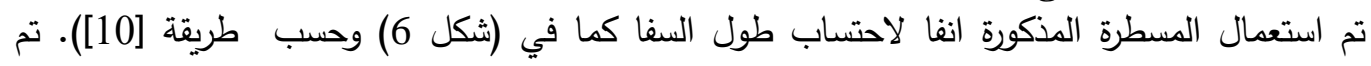
استعمال المهر الضوئي ذي قوة تكبير ×20 مرة لملاحظة كثافة الثعيرات على الجزء القاعدي للعصيفة كما في (شكل 5) وتم تصنيفها كمايأتي:

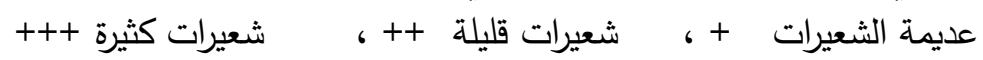
حامل السنابل

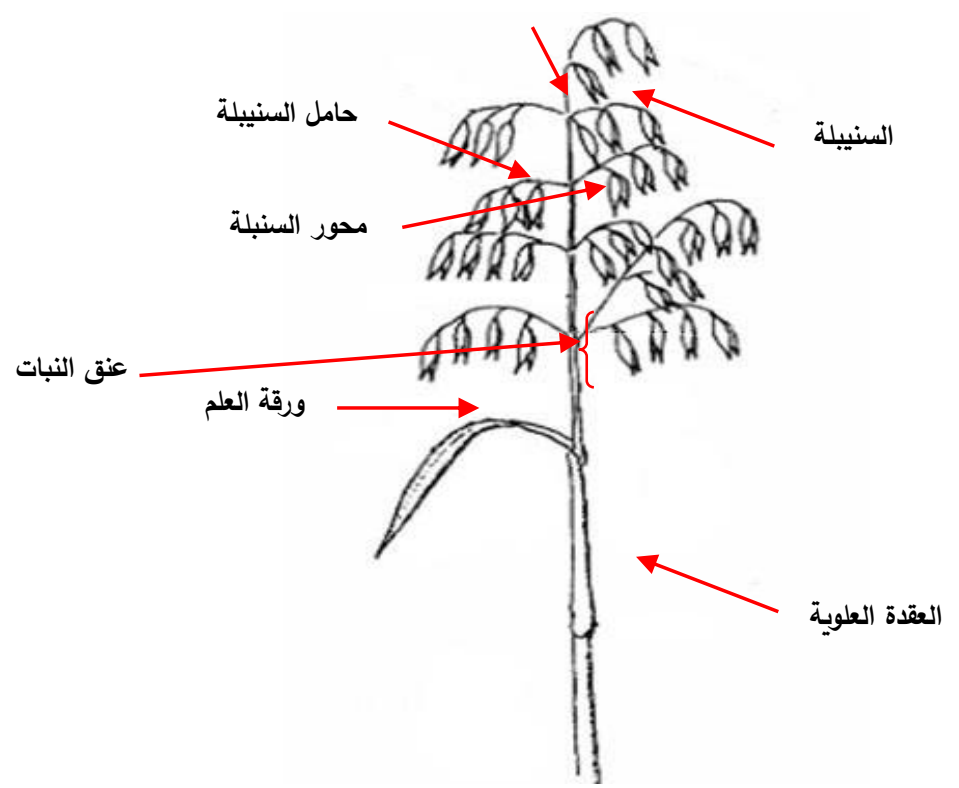

شكل (2): اجزاء نبات الشوفان

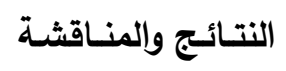

بعد تحليل نتائج هذه الدراسـة إحصـائيا تبين وجود فروقات معنويـة بين الاصناف الزراعيـة في جميع الصفات المظهرية للنبات والتي شملت:

Rachis length and shape طول محور السنبلة وشكلهُ

يشير (الجدول 1) الى وجود اختلافات معنويـة بين الاصناف الزراعية الخمسة في صفة محور السنبلة

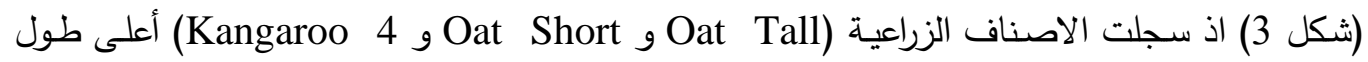
لمحور السنبلة (15.03 , 13.51 , 13.35 سم) على التوالي، ممـا جعلهم مجموعـة متميزة عن بقيـة فئة

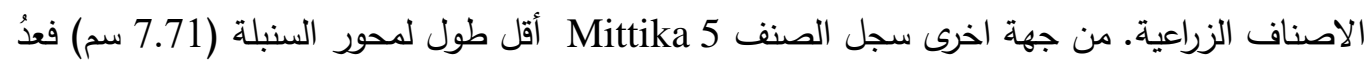
مجموعة مستقلة عن بقية الاصناف الزراعية. أما صنف Possum 6 مكان طول محور السنبلة متوسط

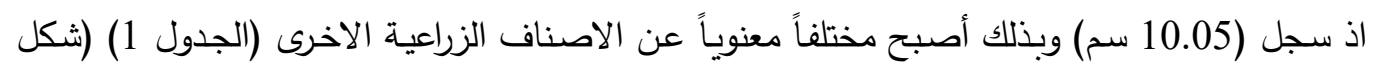

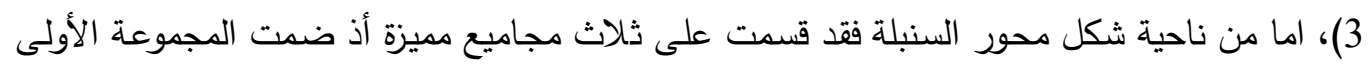




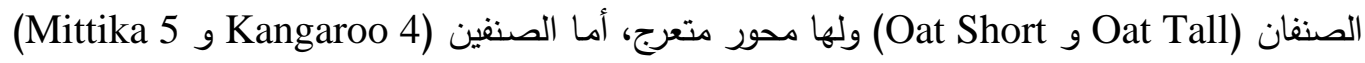
فلها محور غير متعرج وبذلك كونا مجموعة ثانية. أما المجموعة الثالثة فقد تم تكوينها بواسطة صنف الثان الثان Possum 6

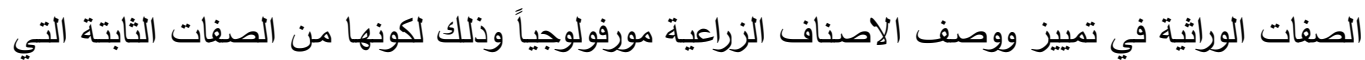

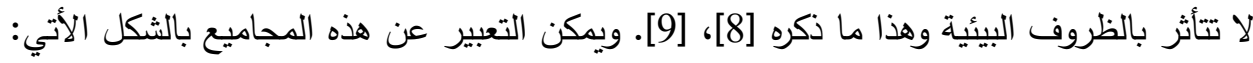

$\underline{\mathrm{M}} \quad \underline{\mathrm{P}} \quad \underline{\mathrm{K}} \quad \mathrm{OS} \quad \mathrm{OT}$

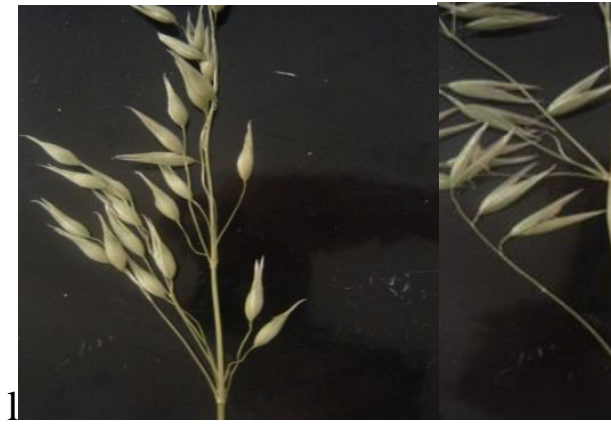

غير متعرج

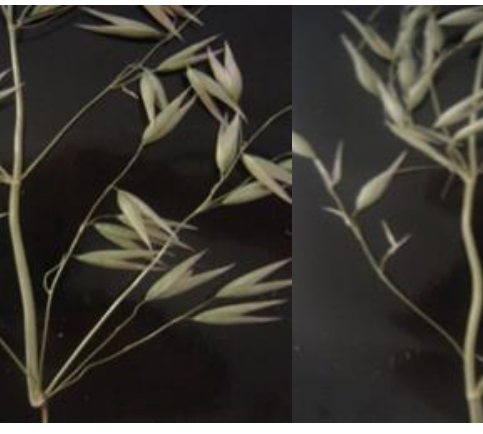

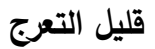

شكل (3): شكل محور السنبلة

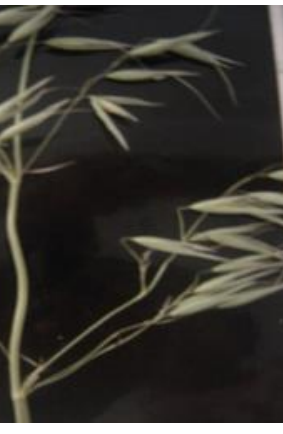

متعرج

جدول (1): معدل طول محور السنبلة، طول حامل السنابل، طول حامل السنيبلة وشكل محور السنبلة في أصناف نبات الثوفان قيد الدراسة.

\begin{tabular}{|c|c|c|c|c|c|}
\hline طول حامل & طول حامل السنابل(سم) & شكل محور & & طول مالسنبلة & الصفات \\
\hline $\begin{array}{ll}\text { ج } & 2.08\end{array}$ & $\begin{array}{ll}\text { ج } & 4.80\end{array}$ & تعرج قليل & ب & 10.05 & Possum 6 \\
\hline 2.28 & 5.69 & غير متعرج & أ & 13.35 & Kangaroo 4 \\
\hline 2.33 & $\begin{array}{ll} & 7.18\end{array}$ & متعرج & أ & 15.03 & Oat Tall \\
\hline ج 2.06 & $\begin{array}{ll} & 3.37\end{array}$ & غير متعرج & ج ( ) ( & 7.71 & Mittika 5 \\
\hline I $\quad 2.52$ & $\begin{array}{ll} & 6.84\end{array}$ & متعرج & i & 13.51 & Oat Short \\
\hline
\end{tabular}

* القيم التي تثترك بنفس الأحرف ضمن العمود الواحد لا تختلف معنويا عند مستوى احتمال 5\% حسب اختبار دنكن.

Peduncle length طول حامل السنابل

عند مقارنة معدلات طول حامل السنابل بين الاصناف الزراعية الخمسة لنبات وجدت فروقات معنويـة عند مستوى 5\% (جدول 1) (شكل 2). وقد تقرقت الاصناف الزراعية إعتمادا على صفة طول حامل السنابل إلى أربعة مجاميع. وقد سجل كل من (Oat Tall و Oat Short)، أعلى طول لحامل السنابل (6.18 و 6.84

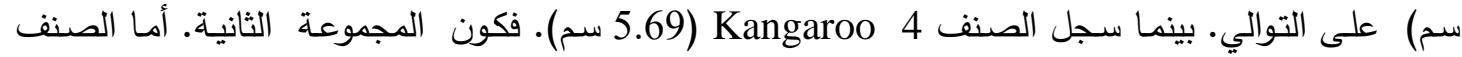
Mittika 5 Possum 6 السنابل وقدرة (3.37 سم) مكونـاً مجموعة رابعة متميزة مختلفة ومستقلة عن بقيـة الاصناف الزراعية الأخرى.

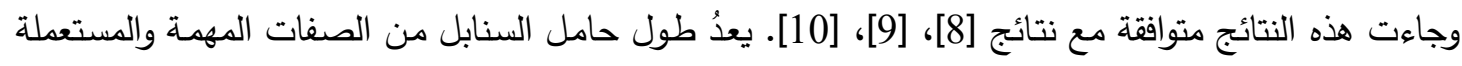
في الدراسات التصنيفية.

ويعبر عن هذه المجاميع كما يلي: $\underline{\text { M }} \quad \underline{\mathrm{P}} \quad \underline{\mathrm{OS}} \quad$ OT


Pedicle length طول حامل السنييلة يبين (الجدول 1) وجود اختلافات معنوية في معدل طول حامل السنيبلة بين أصناف الشوفان المشمولة في هذه الدراسة (شكل 2). وقد تبين ان صنف Oat Short والذي بلغ معدل طول حامل السنيبلة (2.52 سم)

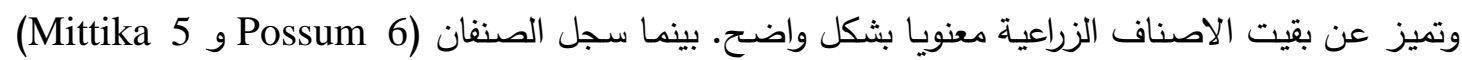
اقصر معدل طول لحامل السنيبلة (2.08 و 2.06 سم) على التوالي. بينما لم نجد اختلافات معنوية في معدل طول حامل السنيبلة بين الصنفين (4angaroo 4 و Oat Tall) أذ سجلا (2.28 و 2.33 سم) على التوالي وكونا مجموعة مستقلة بذاتها. وقد اعتمد كل من [11]، [4]، [10] على صفة طول حامل السنيبلة في دراساتهم التصنيفية. ويمكن التعبير عن الاختلافات بين الاصناف الزراعية في صفة طول محور السنيبلة وعند مستوى

$$
\begin{aligned}
& \text { احتمال 5\% وكما يلي. } \\
& \begin{array}{lllll}
\mathrm{M} & \mathrm{P} & \underline{\mathrm{K}} & \mathrm{OT} & \underline{\mathrm{OS}}
\end{array}
\end{aligned}
$$

Arrangement of spike on the Rachis ترتيب السنابل على محور السنبلة يبين (الجدول 2) وجود اختلافات معنوية في ترتيب السنابل على محور السنبلة بين أصناف الشوفان المشمولة في هذه الدراسة. وقد تميز الصنفان (Oat Short Possum 6) بالثكل السواري في ترتيب السنابل

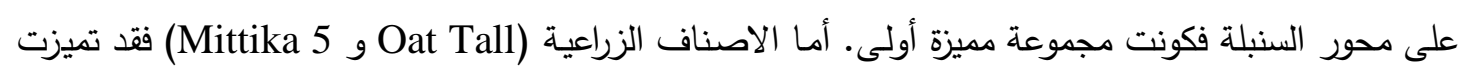
في ترتيب السنابل على محور السنبلة بالثكل المتقابل فكونت مجموعة اخرى. بينما تميز الصنف

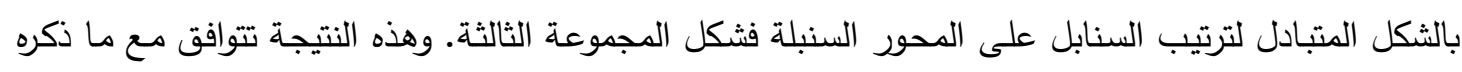

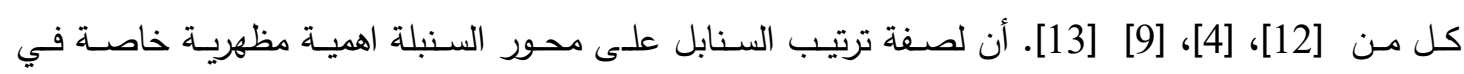
الدراسات التصنيفية اذ انها تعد من الصفات الوراثية والمهمة والثابتة. جدول (2): هيأة ترتيب السنابل على محور السنبلة، متوسط ارتفاع اللسين وشكله في الاصناف الزراعية المشمولة في

\begin{tabular}{|c|c|c|c|c|}
\hline شكل أللسين & & $\begin{array}{c}\text { ارتفاع أللسين } \\
\text { (ملم) }\end{array}$ & ترتيب السنابل على محور & الصفات \\
\hline مثلث & $\overline{1}$ & 0.52 & سواري & Possum 6 \\
\hline شريطي & $\rightarrow$ & 0.35 & متبادل & Kangaroo 4 \\
\hline دائري & أب & 0.46 & متقابل & Oat Tall \\
\hline 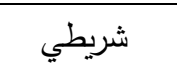 & $\sim$ & 0.36 & متقابل & Mittika 5 \\
\hline دائري & ب ح & 0.42 & سواري & Oat Short \\
\hline
\end{tabular}
هذه الاراسة لنبات الثوفان. * القيم التي تشترك بنفس الأحرف ضمن العمود الواحد لا تختلف معنويا عند مستوى احتمال 5\% حسب اختبار دنكن.

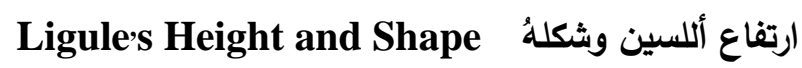
أللسين غشاء رقيق ابيض اللون يقع في منطقة اتصال الغدد مع نصل الورقة. ولصفة ارتفاع أللسين أهمية تصنيفية كبيرة وفي التوصيف المظهري أذ تعدُ من الصفات المورفولوجية الرئيسة للنبات. تثير النتائج في لفي

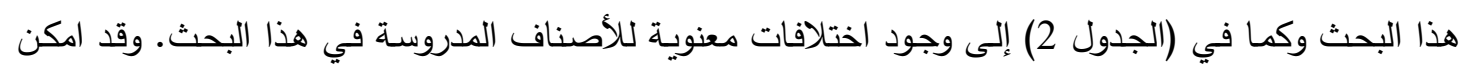
تقسيمها الى عدة مجاميع مختلفة معنويا في صفة ارتفاع اللسين (شكل 4). فقد تم تسجيل أعلى ارتفاع للسين في 


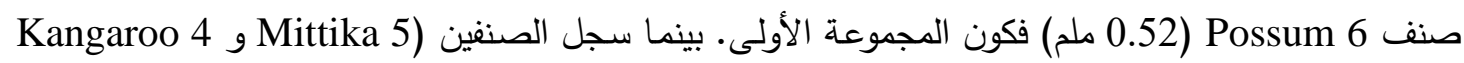

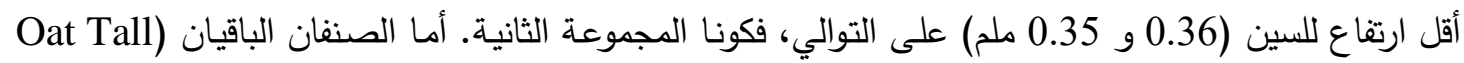
و Oat Short) وقد سجلا ارتفاعاً للسين قدره (0.46 و 0.42 ملم) على التوالي فقد اشتركا بين المجموعتين الأولى والثانية.

اما شكل اللسين فقد وجد اختلافات معنوية بشكل اللسين ويوضح (الجدول 2) الاشكال المختلفة للسين. وقد تميز الصنفان (Oat Short Oat Tall) بالشكل الدائري للسين (شكل 4). وتميز اللسين في نباتات

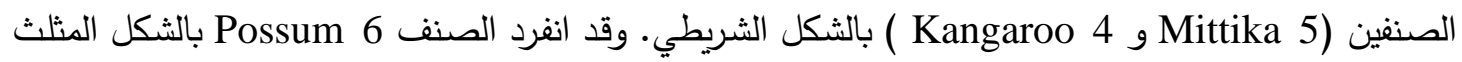
للسين. وهذه الصفة تعدُ من الصفات المورفولوجيـة الثابتة والمهيـة لأنها لا تتأثر بـالظروف البيائية المحيطـة

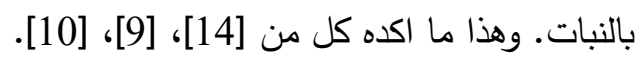
ويمكن التعبير عن الاختلافات بين الاصناف الزراعية باستعمال صفة ارتفاع اللسين بالشكل التالي: OS OT

K M OS OT P

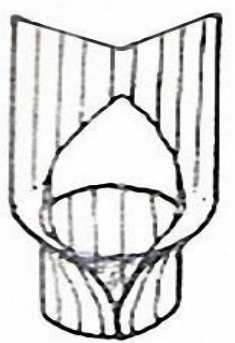

مثtث

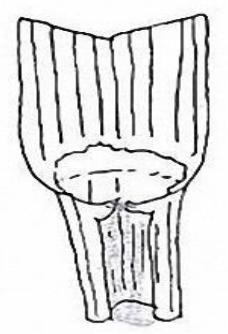

شريطي

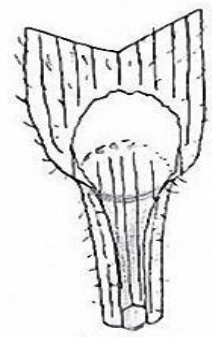

دائري

شكل (4): أشكال أللسين في نبات الثوفان

Glumes Length ول القنابع


للقنابع في اصناف نبات الشوفان المشمولة في هذه الدراسة أذ كانت أطوالها (2.56 , 2.34 سم) على التوالي، وبذلك فقد شكلت مجموعة متميزة بشكل معنوي واضـح عن بقية الاصناف الزراعية الاخرى ذات القنابع الطويلة

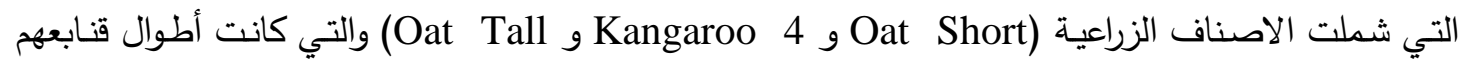

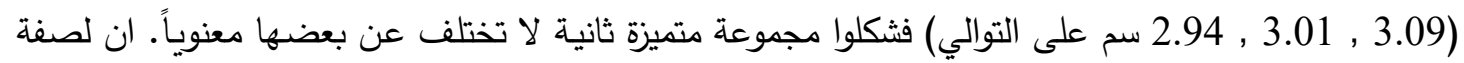

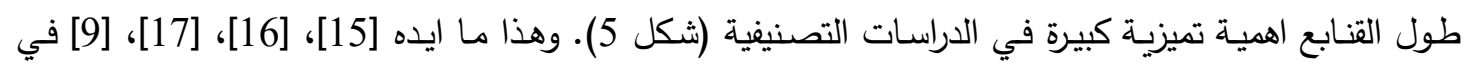

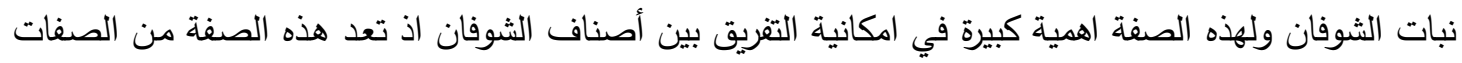


الاصناف الزراعية في صفة طول القنابع هي اختلافات وراثية. ويعبر عن الاختلافات بين هذه المجاميع في صفة طول القنابع بالشكل التالي:

$\underline{\mathrm{M}} \mathrm{P} \quad \mathrm{OT} \quad \mathrm{K} \quad \mathrm{OS}$ 
جدول (3): متوسطات طول القنابع، وطول السفا، وكمية الثعيرات على الجزء القاعدي للعصيفة في أصناف نبات الثوفان المشمولة في هذه الدراسة.

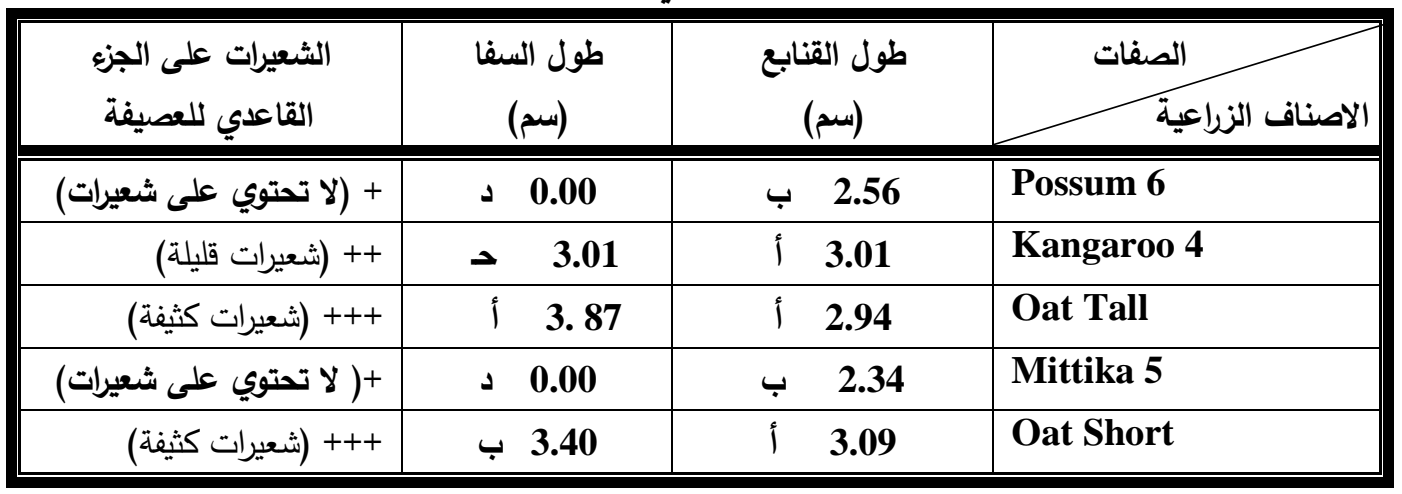

* القيم التي تشترك بنس الأحرف ضمن العمود الواحد لا تختلف معنويا عند مستوى احتمال 5\% حسب اختبار دنكن.

\section{Awn Length}

طول السفا

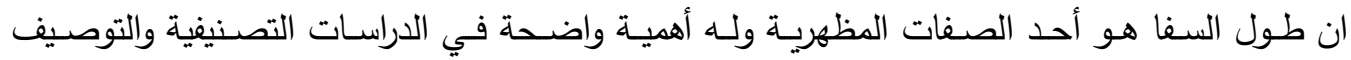
المظهري حاله كحال الصفات الثكلية الأخرى للنبتات، وتدل النتائج الدذكورة في (الجدول 3) إلى أن اصناف الفهاف

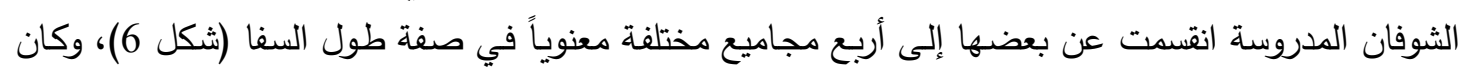

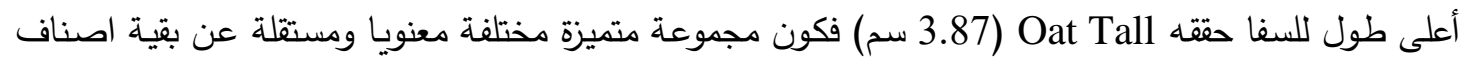
الثوفان. وضمت المجموعة الثانية صنف Oat Short S.40 سم)، وبذلك شكلت المجموعـة الثانيـة. أمـا المجموعة الثالثة فقد حققها الصنف Kangaroo 4 (3.01 سم) فكان مجموعة مستقلة. واخيرا فإن المجموعة الرابعة قد تحققت من قبل الصنفين (Mittika 5 Possum 6 و الذ لم يلاحظ فروقات معنوية بينهما في صفة

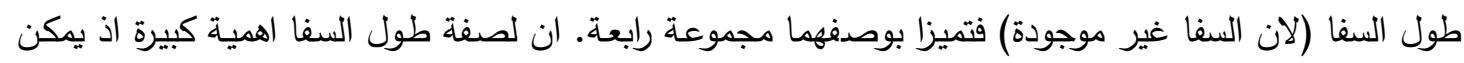

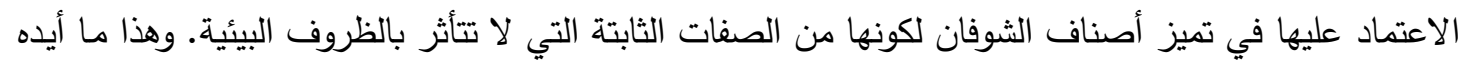

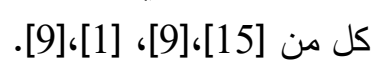

ويعبر عن الاختلافات بين هذه الاصناف الزراعية في صفة طول السفا بالثكل كما يلي:

$\underline{M} \quad \underline{\mathrm{K}} \quad \underline{\mathrm{OS}} \quad \underline{\mathrm{OT}}$

Hairs on the basic part of the Lemma الثعيرات على الجزء القاعدي للعصيفة

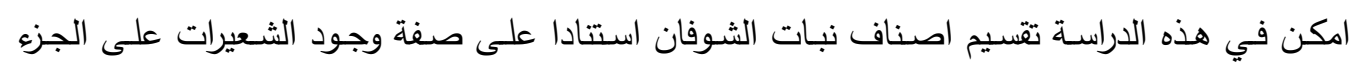


الاصناف الزراعية المدروسة ، فتميزت نباتات الصنفين (Mittika 5 Possum 6) بعدم احتواءها ها على شعيل فيرات على الجزء القاعدي للعصيفة فكونا مجموعة واحدة. في حين أن نباتات الصنفين (Oat Short, Oat Tall) اشتركا مع بعضهما في صفة وجود شعيرات كثيفة على الجزء القاعدي للعصيفة واحتسبا مجموعة مستقلة ثانية.

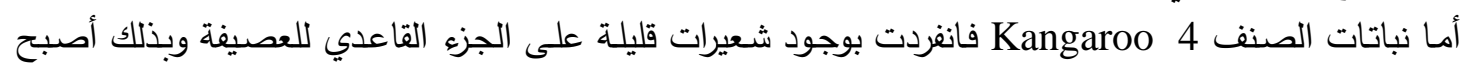

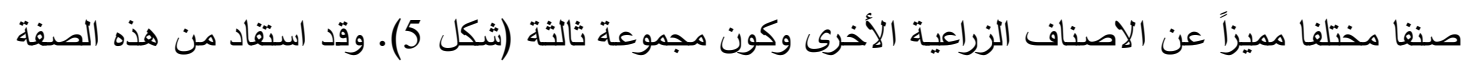

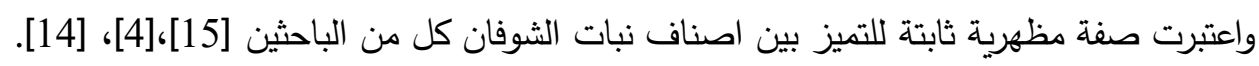




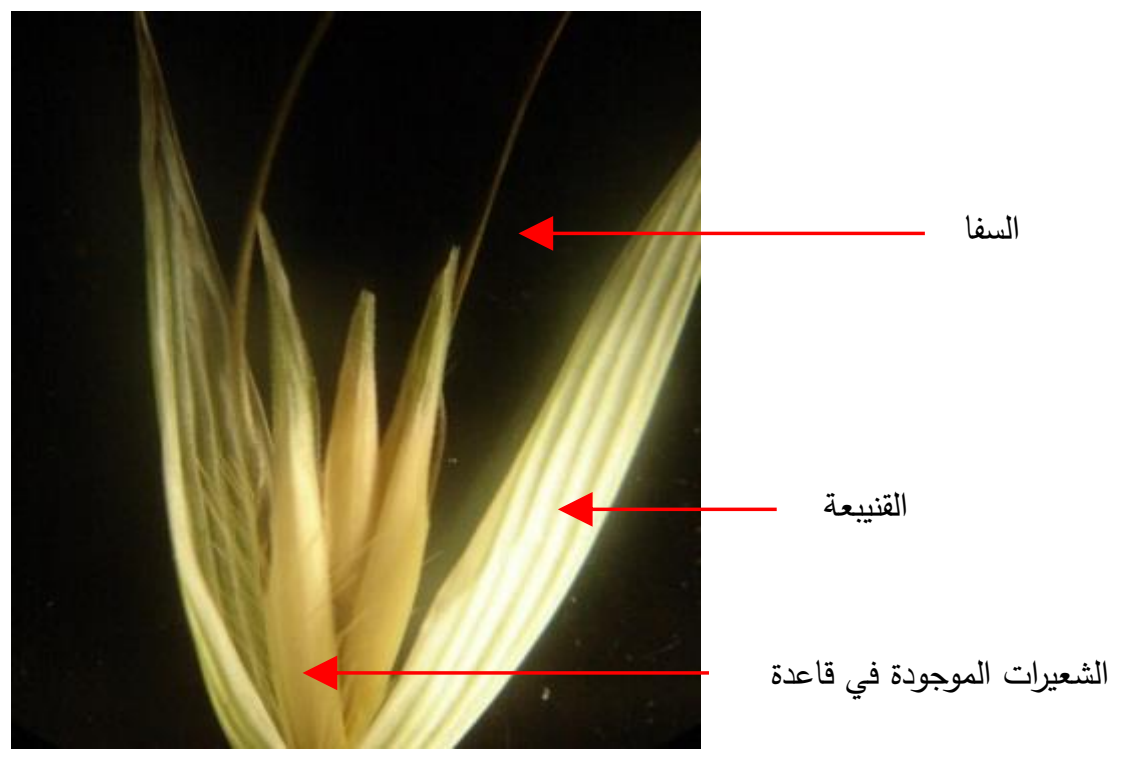

شكل (5): القنابع والعصيفات في نبات الثوفان

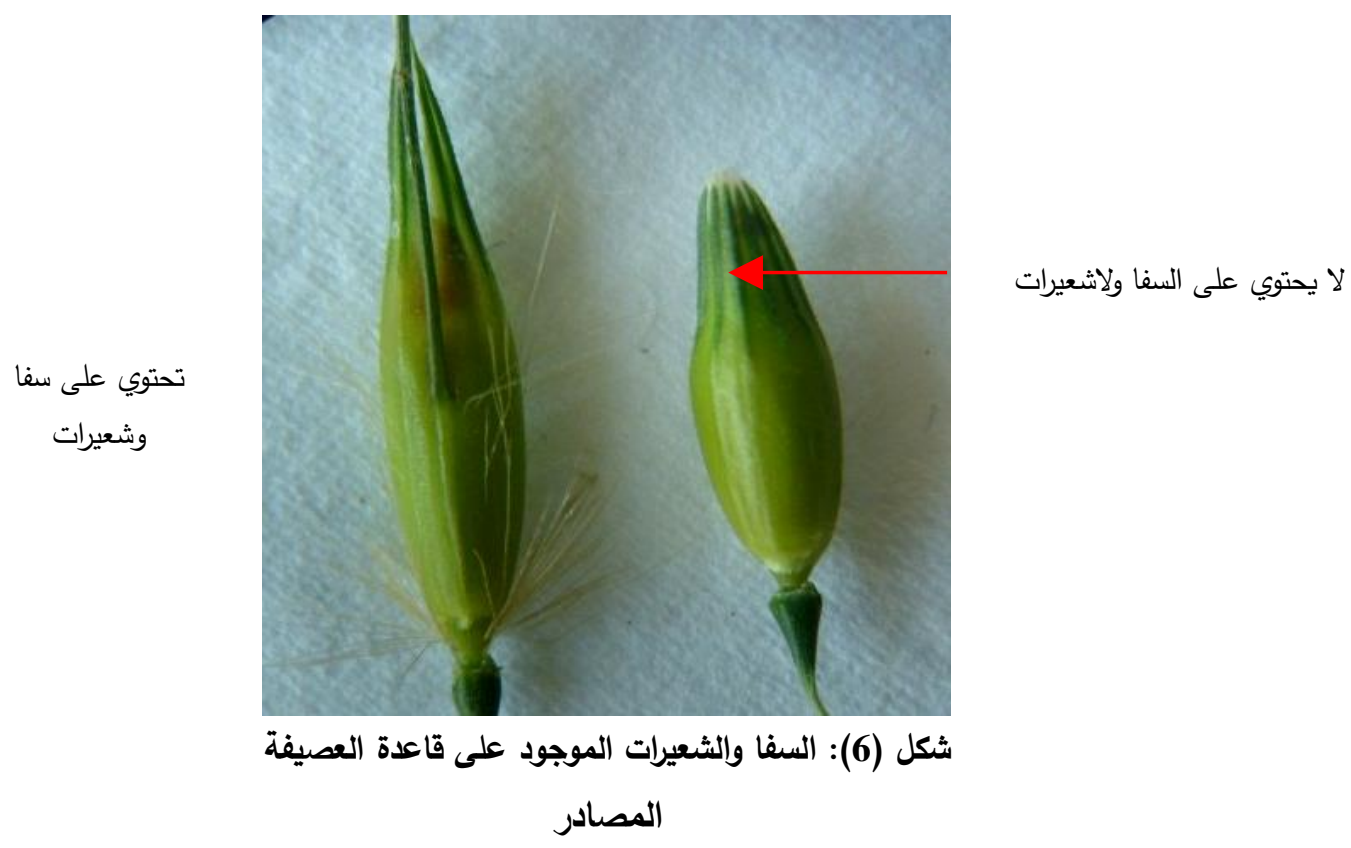

1) Mahmud R.H., Morphological characteristics of grains and plants in of some types of wheat Triticum sp. Master Thesis, Faculty of Education, University of Mosul (2004). (In Arabic)

2) Chakravarty HL. Plant wealth of Iraq. (A dictionary oF Economic plants). Vol 1; Botany Directorate Bagdad. Ministry (1976).

3) Coffman FA. Oat History: Identification and classification. Techn. Bulet. 1516 1525 (1977).

4) AL. Younis A.A, Mahfuz A., Zaki A. product grain, Dar Al Kutb for Printing and Publishing / University of Mosul, PO Box 333-345 (1987)

5) Li CD, ROSsnagel BG, Scoles GJ. Tracing The phylogeny of the Hexaploid Oat Avena sativa with satellite DNAs. Crop Sci. Soc. Amer. 40: 1755 - 1763 (2000).

6) Alkatib, Y.M. Classification of seed plants. Second edition, Dar al-Kitab for printing and publishing, University of Baghdad (2000) 
7) Amin H M, Ali A.H. Examination and certification of seeds, Ministry of Higher Education and Scientific Research. University of Baghdad (1988).

8) Sheidai M, Koobaz $\mathrm{P}$, Termeh $\mathrm{F}$, and Zehzad B Phenetic studies in Avena species and populations of Iran. J. Sci., 13(1): 19-28(2002) .

9) CFIA (Canadian Food Inspection Agency) Seed progra. specific work instructions. Cereal crop inspection procedures 1-21(2005).

10) Gibson DJ Grasses and Grassland and Ecology. $1^{\text {st }}$ ed; oxford, UK, pp: $35-42$ (2009).

11) Duke JA . Avena sativa L., Handbook of Energy crops. Center for New crops and plants products. purdue University pp: $1-7(1983)$.

12) Magness AI .Oat (Avena sp.). Center for New Crops and plant of products, Purdue University 3: 1-4(1971).

13) Ahmad G, Ansar M, Kaleem S, Nab G, Husain M. Performance of early maturing Oat (Avena Sativa) cultivars for yield quality. J. Agric. Res. 6: 341-346 (2008).

14) Sauerborn E, Sauerborn J Weeds of West Asia with special reference to Syria. PIITS. 6(1): 357-359(1988).

15) Townsend CC, Guest E, AL-Raw A Flora of Iraq. Volume 9; Ministry of agaiculture, Bagdad, Repulic of Iraq. pp: 327-344 (1968).

16) Ougham HJ, Latipova G, Valentine J. Morphological and biochemical characterization of spikelet development in naked Oats (Avena sativa). New Phytolo. 134 (1): 5-1(1996)

17) Suttie JM Avena sativa L. FAO. 67: 1-4(2002).

18) Baum BR, Bailey LG Toxonomy of the north and south American species of the Hordeum section Hordeastrum. Can. J. BOT. vol. 60 (8): 1745-1759(1986).

19) Mohammed M.A. Distinguishing between some barley varieties Hordeum sp. Using the morphological characteristics of seeds and plants. Master Thesis, Faculty of Education, University of Mosul. (1999) (In Arabic). 